\title{
Ant Colony Intelligence in Flexible Manufacturing Scheduling Using Contract-Net Model
}

\author{
Yingzi Wei ${ }^{1,2, a}$, Yijun Feng ${ }^{1}$, Kanfeng $\mathrm{Gu}^{2}$ \\ ${ }^{1}$ Shenyang Ligong University, Shenyang 110159, China \\ ${ }^{2}$ Shenyang Institute of Automation, Chinese Academy of Science, Shenyang 110016, China \\ awings_syit@126.com
}

\begin{abstract}
Key words: Flexible manufacturing scheduling, Ant colony intelligence, Contract-net protocol, Multi-agent system
\end{abstract}

\begin{abstract}
This paper builds an efficient agent-based flexible scheduling for real-world manufacturing systems. Considering the alternative processes and alternative machines, the allocation of manufacturing resources is achieved through negotiation among the job and machine agents in a multi-agent system (MAS). Ant Colony Intelligence (ACI) is proposed to be combined with Contract Net Protocol (CNP) so as to make agents adaptive to changing circumstances. ACI is integrated into both machine agents and job agents to solve the task allocation and sequencing problem. CNP is introduced to allow the agents to cooperate and coordinate their local schedules in order to find globally near-optimal robust schedules. The negotiation protocol is an interactive bidding mechanism based on the hybrid contract net protocol. The implementation of the issues using CNP model is discussed. Experimental results verify the effectiveness and efficiency of the proposed algorithm integrated with ant-inspired coordination.
\end{abstract}

\section{Introduction}

Flexible job-shop scheduling problem (FJSP) is an extension of the classical job-shop scheduling problem (JSP) which allows an operation to be processed by any machine from a given set. This study aims at building an efficient agent-based flexible scheduling model for real-world manufacturing systems with various products and processes. In consideration of the alternative processes and alternative machines, the actual selection of the schedule and allocation of manufacturing resources is achieved through negotiation among the job and machine agents in a multi-agent system.

A MAS not only provides parallel execution of commands but also has the intelligence of negotiation to enhance system performance. A hybrid algorithm is developed to support MAS architectures. This new negotiation protocol enables multi-task many-to-many negotiations, where global control is incorporated into the decentralized negotiation. In this work, ACI is proposed to be combined with local agent coordination so as to make autonomous agents to give rise to efficient global performance.

The structure of this paper is as follows: in section 2, the ant colony intelligence, especially ant colony intelligence-based approach in flexible manufacturing system is presented. The definition of contract net protocol and the combination of ACI and CNP is given in section 3. Section 4 presents a case study of FJSP, also the results analysis and discussion are given. Finally, conclusions and ideas for future work are presented in section 5.

\section{Ant colony intelligence in flexible job shop scheduling}

Dorigo et al. firstly introduced Ant Colony Optimization (ACO) for solving the TSP which is based on ant foraging. Later, ACO was successfully applied to many other combinatorial optimization problems, including manufacturing scheduling problems. However, most of ACO applications in scheduling deal with static problems. For dynamic manufacturing problem, Cicirello and Smith 
introduced ant colony control for shop floor routing in a dynamic factory setting. Ants are assigned to new jobs, and all communication is accomplished indirectly through the real-time changing process of updating pheromone quantities located at the various machines. The limitation in this system is that it fails to adapt appropriately to changing job mixes once it is converged to specialized product lines for multiple job types and multi-purpose machines.

This study aims at building an efficient agent-based flexible scheduling for real-world manufacturing systems with various products and processes. ACI is proposed to be combined with local agent coordination so as to make autonomous agents adaptive to changing circumstances and to give rise to efficient global performance. The study here differs from other flexible scheduling research in two areas: (1) a more generic and realistic manufacturing model with multiple product types, parallel multi-purpose machines with sequence-dependent setup constraints. (2) ACI integrated with both machine agents and job agents to solve not only the task allocation problem, but also the task sequencing problem. The implementation of the aforementioned issues in a multi-agent system (MAS) is discussed. Simulation results show that, for most of the performance measures, a MAS integrated with well-designed ant-inspired coordination performs well compared to a MAS using dispatching rules.

\section{A contract net protocol based approach using ACO}

CNP is a protocol for matching up tasks with agents in multi-agent stem [1]. CNP and its variants are probably the most widely used agent system protocol, presumable because of its intuitiveness, direct applicability to many common problems, simplicity and relative efficiency. CNP has been applied to many domains including manufacturing control [2], tactical simulations [3], transportation scheduling [4], and distributed sensing, CNP is also an abstract version of the one-round-sealed-bid auction protocol used in a number of today's B2B exchanges.

In a basic CNP [5], one agent announces the availability of tasks, broadcasts them to other bidding agents, selects a contractor, and finally awards the task by comparing bids corresponding to a particular offer using predefined criteria. Like previous work [6], cooperative negotiation is achieved as following:

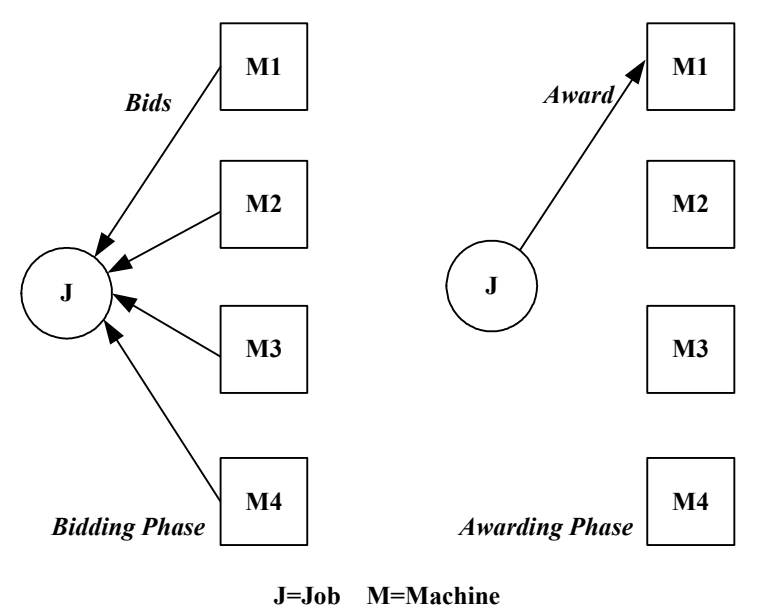

Fig. 1. Job-centered bidding perspective

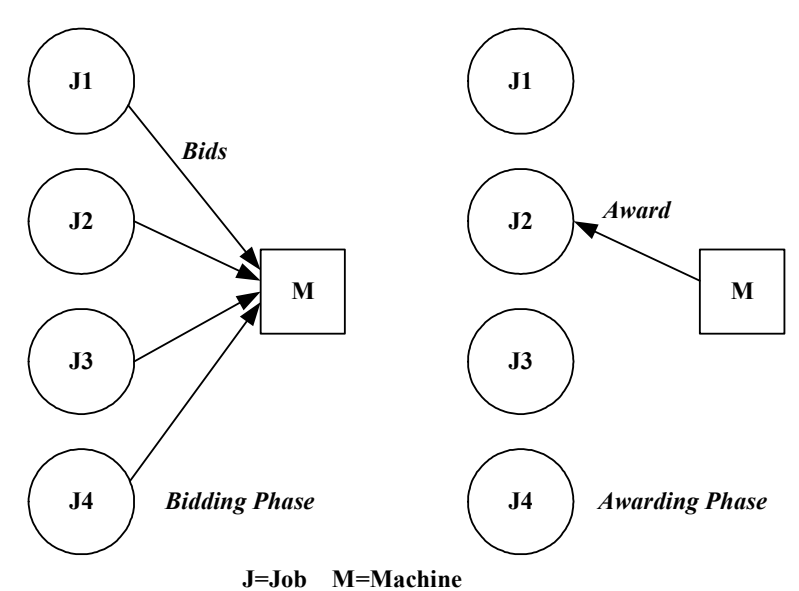

Fig. 2. Machine-centered bidding perspective

The negotiation protocol is an interactive bidding mechanism based on the hybrid contract net protocol. Job-centered negotiation is machine-initiated bidding; machines make bids and compete with each other to strive for a job processing (shown in Fig. 1). In making its bid, a machine attempts to optimize its own performance measures, such as keeping its utilization within a desired interval and making its buffer levels (commonly referred to as work-in-progress, in short, WIP) low. The job is awarded to the machine offering the highest bid. Machines use this scheme to bid for 
jobs and pull them through the manufacturing system. Machine-centered negotiation is job-initiated bidding, i.e., job agents make bids to the machines comprising the manufacturing system (shown in Fig. 2). The targets of the bidding are the services or operations offered by the machines. A job's bid should reflect its own criteria for being processed, such as its due date and priority. The higher a job's bid, the more attractive and possible it should be for the machine to select the job for processing. In this scheme, jobs negotiate with the machines to push themselves through the manufacturing system. ACI is integrated into agent coordination for both the machine agent and the job agent. It helps the job agent to find an appropriate machine agent for processing, and also helps the machine agent to determine the next job to be processed in the current queue. Here, each machine agent is regarded as an ant. Also, each job agent is considered as an ant. The pheromones for the machine agent and job agent both have special definitions, respectively.

ACI in machine agent. Here, each machine agent is regarded as an ant, and the pheromone for the machine agent is defined as following. The machine with the shortest processing time (SPT) for its associated operation will have the highest initial pheromone

$$
\tau_{M A_{i}}(0)=\frac{\sum_{j} \sum_{l} \eta_{j l}^{i}}{\sum_{j} \sum_{k} \sum_{l} \eta_{j l}^{k}}
$$

where

$$
\eta_{j l}^{k}=\frac{1}{P T_{O_{j l}}^{k}}
$$

Operation $O_{j l}$ is the $l$ th operation of job $j, P T_{O_{j l}}^{k}$ represents the processing time of $O_{j l}$ on machine $\mathrm{MA}_{i}$, and $\eta_{j l}^{k}$ is the heuristic information associated with operation $O_{j l}$.

The pheromone for a machine depends on two aspects, one is the local status of the machine agent, the other is the total waiting processing time of the machine entity

$$
\tau_{M A_{i}}(t)=\frac{x_{M A_{i}(t)}}{\sum_{j} \sum_{l} q_{j l}^{i}(t) P T_{j l}^{i}}
$$

where $x_{M A_{i}(t)}$ is the local status of machine agent $\mathrm{MA}_{i .} x_{M A_{i}(t)}=1$ means the machine is in good working state, either "busy" or "idle", $x_{M A_{i}(t)}=0$ signifies the machine is in the "buffered" state. $q_{j l}^{i}(t)$ indicates the operation's queue status. $q_{j l}^{i}(t)=1$ implies operation $O_{j l}$. is in the queue of $\mathrm{MA}_{i}$, otherwise, $q_{j l}^{i}(t)=0$. The probability of which a job agent selects machine agent for processing an operation is dependent on the amount of the machine's pheromone and the heuristic information associated with operation

$$
p\left(M A_{I}\right)=\frac{\left(\tau_{M A_{i}}\right)^{\alpha}\left(\eta_{j l}^{i}\right)^{\beta}}{\sum_{k}\left(\tau_{M A_{i}}\right)^{\alpha}\left(\eta_{j l}^{i}\right)^{\beta}}
$$

where $\alpha$ and $\beta$ are the tuning parameters for balance the effects of pheromone and heuristics, and can be achieved by simulation. The machine agent with the greater transition probability will have a greater chance to be selected by the job agent. 
ACI in job agent. The job agent's pheromone is important for the task sequencing problem. Initially, the individual job agent's pheromone depends on the due date tightness of the associated job entity. The job agent with highest due date of its job will have the highest initial pheromone

$$
\tau_{J A_{i}}(0)=e^{-\left(D u e_{J A_{i}}-t_{0} / \sum_{l} P T_{O_{i l}}\right)}
$$

where $D u e_{J A_{i}}$ and $\sum_{l} P T_{O_{i l}}$ are the due date and the total processing time of job $i$, respectively. Due date is computed by the following formula

$$
\text { Due }=\text { Arriva }+k \sum_{i=1}^{n} P T_{i} \quad k \in[2,10]
$$

Later, when the job has been processed, the pheromone value is determined by the remaining time before the due date and the remaining processing time of the job

$$
\tau J A_{i}(t)=e^{-\left(D u e_{J A_{i}}-t / \sum_{l} f_{O_{i l}}(t) P T_{O_{i l}}\right)}
$$

where $f_{O_{i l}}(t)$ is the finish status of the operation. $f_{O_{i l}}(t)=0$ implies the operation $\mathrm{O}_{i l}$ is finished, $f_{O_{i l}}(t)=1$ implies the operation is not yet finished. Eq (7) shows that the job agent's pheromone is a function of time, it should be updated continuously.

The probability, with which a machine agent selects operation $\mathrm{O}_{i l}$ of job agent $\mathrm{JA}_{i}$ as the next processing operation from the current queue is dependent on the amount of a job's pheromone, the heuristic information associated with the operation $\mathrm{O}_{i l}$ as well

$$
p\left(J A_{i}\right)=\frac{\left(\tau J A_{i}\right)^{\alpha}\left(\eta_{i l}^{i}\right)^{\beta}}{\sum_{k}\left(\tau J A_{k}\right)^{\alpha}\left(\eta_{k l}^{k}\right)^{\beta}}
$$

The job agent with greater transition probability will have a greater chance to be selected by the machine agent as their next processing job in the current queue.

Agent status. Each agent has a life cycle in a MAS, so agent shows different states during its life cycle. Each agent has both global and local states which describing the general agent state and associated entity's state, respectively. The global status includes the following states (1) initiated, (2) suspend, (3) active, (4) deleted, and (5) waiting, which determine different agents activities. Such global states are generally the same in each agent in a MAS. The local status is more focused on entity states; therefore, it is entity-specific and is different according to agent types.

The statistic of physical entity in manufacturing system can be analyzed easily by tracing the individual agent's local status. For example, as long as a machine agent registers with a shop floor, it starts in the "idle" state, then it turns to the "busy" state when an operation of job starts a process on an associated machine; at this moment, any further incoming jobs will be placed in a queue and the machine agent changes to the "buffered" state as long as there exists operations in the queue; after the machine finished it's task, it turns to the "idle" state again, and starts to bid for operations. Hence, with these defined local states, the associated machine's statistics analysis can be easily monitored and calculated. 


\section{Case Study}

To investigate the impact of integrating ACI in agent coordination, a multi-agent manufacturing system is built up for simulating a realistic shop. In this section an example about the FJSP [7] is introduced and solved by the proposed approach. Table 1 lists the information for this problem. This example has four jobs with 11 operations, 3 machines shown in table 1 . The process time for the capable machines of each operation is also shown in this table. At first, the problem is solved by the mathematical method and its results are shown in Fig.3 [7].

Fig. 4 shows the result of the proposed algorithm for problem in table 1. The routing is different from the previous result obtained by mathematical method. Our algorithm found the optimal makespan of 16 days same as the result of previous mathematical method. The total processing time and utility of machines made some improvement, too. The performance of the proposed algorithm is compared with the mathematical method in table 2 . As shown in this table, the total process time obtained by the proposed algorithm is 42 days, 2 days shorter than the result of the mathematical method. Also the utility of machines are higher than the previous results. Due to the introduction of ant colony pheromone in both machine agent and job agent, our algorithm is not only capable of achieving the optimal solution for the example, but also improves the utilization of the machines.

Table 1 An example for FJSP

\begin{tabular}{|c|c|c|c|c|c|c|}
\hline \multirow[t]{3}{*}{ Jobs } & \multicolumn{6}{|c|}{ Operations } \\
\hline & \multicolumn{2}{|l|}{1} & \multicolumn{2}{|l|}{2} & \multicolumn{2}{|l|}{3} \\
\hline & \multicolumn{2}{|c|}{ Machine no. Process time(day) } & \multicolumn{2}{|c|}{ Machine no. Process time(day) } & \multirow{2}{*}{$\frac{\text { Machine no. }}{1}$} & \multirow{2}{*}{$\frac{\text { Process time(day) }}{2}$} \\
\hline \multirow[t]{4}{*}{ Job1 } & 1 & 8 & 1 & 6 & & \\
\hline & 2 & 5 & 2 & 5 & 3 & 4 \\
\hline & & & 3 & 2 & & \\
\hline & & & 4 & 7 & & \\
\hline \multirow[t]{3}{*}{ Job2 } & 2 & 2 & 2 & 7 & & \\
\hline & 3 & 5 & 4 & 4 & & \\
\hline & 4 & 3 & & & & \\
\hline \multirow[t]{2}{*}{ Job3 } & 1 & 8 & 2 & 7 & 3 & 8 \\
\hline & & & 4 & 2 & 4 & 5 \\
\hline \multirow[t]{4}{*}{ Job4 } & 1 & 5 & 2 & 2 & 1 & 2 \\
\hline & 2 & 7 & 3 & 4 & 2 & 3 \\
\hline & & & & & 3 & 7 \\
\hline & & & & & 4 & 5 \\
\hline
\end{tabular}

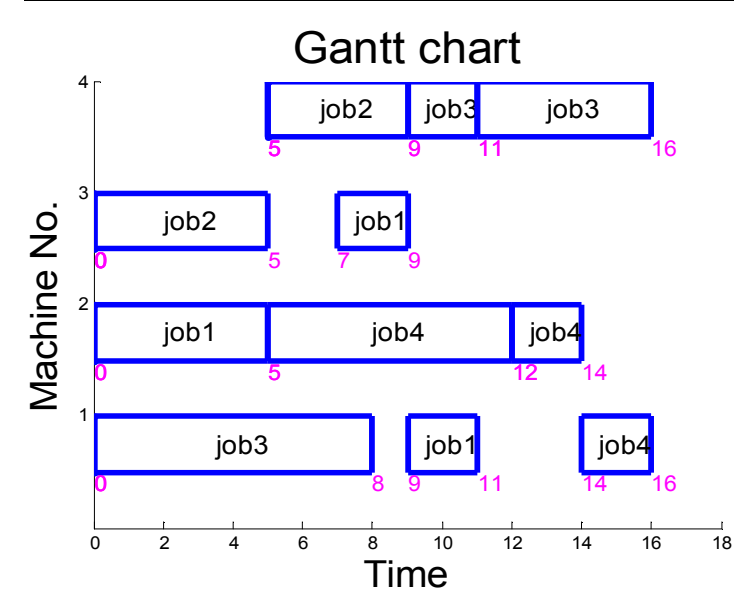

Fig.3.The solution solved by mathematical method [7]

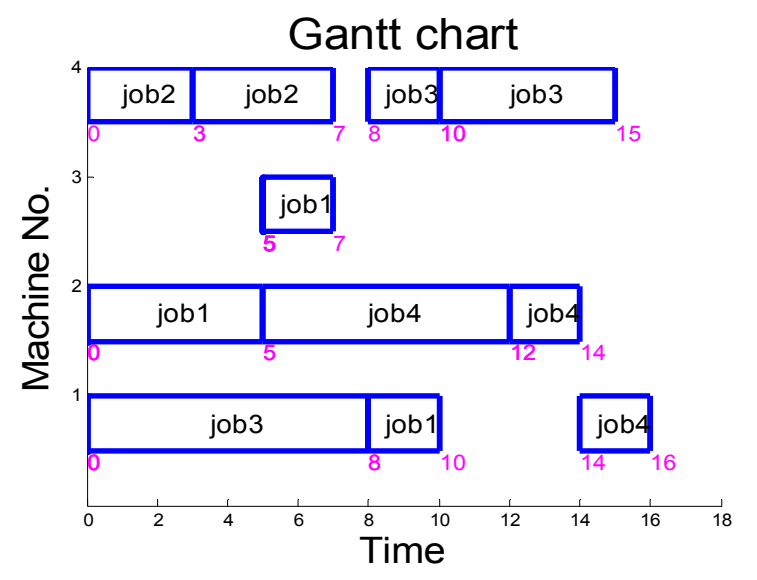

Fig.4.The solution solved by our algorithm 
Table 2 The comparison of performance measure with different methods

\begin{tabular}{|c|c|c|c|c|c|c|c|}
\hline & Makespan & $\begin{array}{c}\text { Total process } \\
\text { time }\end{array}$ & $\begin{array}{c}\text { Utility of } \\
\text { M1 }\end{array}$ & $\begin{array}{c}\text { Utility of } \\
\text { M2 }\end{array}$ & $\begin{array}{c}\text { Utility of } \\
\text { M3 }\end{array}$ & $\begin{array}{c}\text { Utility of } \\
\text { M4 }\end{array}$ & Total utility \\
\hline $\begin{array}{c}\text { Math } \\
\text { method [7] }\end{array}$ & 16 & 44 & $12 / 16=0.75$ & 1 & $7 / 9=0.78$ & 1 & $44 / 55=0.8$ \\
\hline $\begin{array}{c}\text { our } \\
\text { algorithm }\end{array}$ & 16 & 42 & $12 / 16=0.75$ & 1 & 1 & $14 / 15=0.93$ & $42 / 52=0.81$ \\
\hline
\end{tabular}

\section{Conclusion and Discussion}

This paper proposes ant colony intelligence based optimization which represents an efficient approach to the scheduling of flexible manufacturing system including alternative machines and routings. ACI combines with contract net protocol so as to make agents adaptive to changing circumstances. The implementation in a MAS is discussed. Experimental results show that, for most of the performance measures, a MAS integrated with well-designed ant-inspired coordination and performs well compared to a MAS using traditional scheduling method. At the same time the combination of ACI and CNP highly improves solution quality. The use of job-centered negotiation and machine-centered negotiation prompts our algorithm to improve the utilization of process machines and achieve the optimal solution for the case of FJSP.

Future study will be focus on two main directions (1) increasing computing speed for real-time response behavior in flexible manufacturing scheduling applications; (2) allowing the system to support dynamic scheduling.

\section{References}

[1] Smith, R.G. (1980) The contract net protocol: high level communication and control in a distributed problem solver. IEEE Transactions on Computers 29 (12):1104-1113

[2] Baker, A. (1998).Complete manufacturing control using a contract net: a simulation study. 1998 International Conference on Computer Integrated Manufacturing. IEEE Comput. Soc. Press, pp. 100-109. Washington, DC, USA.

[3] Boettcher, K, D. Perschbacher, et al. (1987), Coordination of distributed agents in tactical situation. IEEE 1987 National Aerospace and Electronics Conference: NAECOM 1987(Cst. No. 87CH2450-5). IEEE, pp, 1421-1426 vol.4 New York, NY, USA

[4] Bouzid, M. and A. Mouaddib (1998), Cooperative uncertain temporal reasoning for distributed transportation scheduling, Proceeding of Third International Conference on Multi Agent System, Paris, France. IEEE Comput.Soc.1998, pp. 397-404

[5] W. Xiang, H. P. Lee, Ant colony intelligence in multi-agent dynamic manufacturing scheduling. Engineering Application of Artificial Intelligence 21(2008)73-85

[6] Saad A., Kawamura K. and Biswas G., Performance Evaluation of Contract Net-Based Heterarchical Scheduling for Flexible Manufacturing Systems, Intelligent Autonomous and Soft Computing, 1997, vol. 3, no. 3, pp. 229-248.

[7] Parviz Fattahi, Alireza Fallahi, Dynamic scheduling in flexible job shop system by considering simultaneously efficiency and stability. CIRP Journal of Manufacturing Science and Technology (2010)114-123 